\title{
Preface to the inaugural issue of Quantum Studies: Mathematics and Foundations
}

\author{
Yakir Aharonov • Fabrizio Colombo • \\ Daniele C. Struppa · Jeff Tollaksen
}

Published online: 22 August 2014

(C) Chapman University 2014

Quantum physics is the most successful scientific theory in history. It has led to a profound understanding of the micro-world. It has resulted in technological advances that drive our society and economy, such as the computer revolution, and electronics, optics and the nuclear power industry. In addition, it impacts many other disciplines such as genetics, medicine and mathematics.

Recently, the physics community celebrated the 100th anniversary of its most famous scientific gathering, the Solvay conference of 1911. Many of the questions asked then remain open until today. While scientists have used quantum theory to calculate basic physical quantities with great precision, the foundations of the theory are counterintuitive to such an extent that there is still no consensus as to the meaning of the theory. This may be impeding the development of new forms of quantum theory required for its extension to new frontiers such as quantum computing, string theory, cosmology, gravity and higher energy particle physics.

The primary goal of this international journal is to promote a deeper understanding of all fundamental aspects of quantum theory. Furthermore, the goal is to build a bridge between theoretical questions, foundational issues, mathematical methods and the further evolution of quantum physics. As can be seen by the contents in this inaugural issue, we have welcomed papers in and between these domains. The emphasis is on mathematical methods and insights that lead to a better understanding of the paradoxical aspects of quantum physics and to its expansion into new domains. We encourage the creative use of such paradoxes.

There have been three fundamental routes to advancing the theoretical basis of quantum mechanics that have proven successful since the founding days of the concept of the quantum:

1. The "bringing together of opposites" was implicit in the way Planck solved the radiation law problem. Bohr took this principle of union of opposites and made it the fundamental principle for the philosophical basis of quantum mechanics. The union of opposites is most clearly illustrated by the complementarity principle where the quantum becomes classical in the limit of large numbers.

2. Paradox, with an accompanying tradition of illustrating it with a thought experiment, was the tool Einstein and Schrödinger used to explore the philosophical and axiomatic basis for the strangeness that lies at the heart of quantum mechanics.

Y. Aharonov · D. C. Struppa · J. Tollaksen $(\varangle)$

Institute for Quantum Studies and Schmid College of Science and Technology, Chapman University, Orange, CA, USA

e-mail: tollakse@chapman.edu

F. Colombo

Politecnico di Milano, Milano, Italy and Institute for Quantum Studies, Chapman University, Orange, CA, USA 
3. Finally, there is the approach championed by Dirac and repeated successfully by Feynman and later by Freeman Dyson, namely "playing with equations" as Dirac puts it. This approach sometimes causes equations to reveal their secrets as in the Dirac equation. Dirac took this approach and created results that mathematicians and physicists are still digesting. Feynman, first with the Lagrangian approach to quantum mechanics, the so-called path integral approach, and later with QED and most of the subsequent papers he wrote, operated in this manner. The same could be said of what Dyson did when he "cleaned up" QED into a methodology usable for calculations. Playing with the problems of quantum mechanics often leads to the creation of new mathematics.

While the second approach, finding the root paradox that illustrates the physical by a thought experiment, has been our team's principal tool for conceptualization, as we have explored both the meaning and underlying physical reality of quantum principles, we have used all of these approaches in our various publications as well. They can provide deep insights into the problems that we have raised and sometimes managed to find solutions to.

Another goal of this journal is to capture the spirit of what these founders did. Quantum mechanics remains an open subject theoretically, experimentally and mathematically. It is important to do away with the advice often given to students when we first studied quantum mechanics: "Don't think, shut up and calculate." We have ignored this advice in our whole career, as have our students and collaborators. So we believe it is important to have a repository for ideas, theories and mathematics for those who choose to ignore this advice. We see this journal as a vehicle to explore, as well as reconsider, the foundations of quantum mechanics. It is equally important to recognize the creative element, as Einstein put it, of physics that lies in mathematics. Modern mathematics journals do not always recognize the explorative, tentative nature of initial attempts to extend the horizons for mathematics that are associated with the physical world. We hope that this journal provides a home for those who think there are new worlds to be discovered by looking deeply into quantum mechanics. Our advice is: "Think, reconsider, explore, create deep questions, use paradoxes as a tool for understanding, and finally: publish in this journal!"

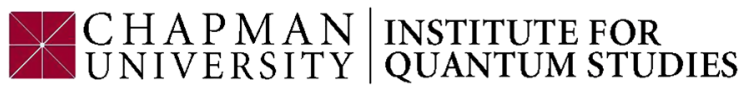

\section{Overview of Volume 1, Issue 1:}

This inaugural issue of the journal deals with some of the most fundamental questions in quantum mechanics including nonlocality, collapse, uncertainty, determinism, measurement and gauge, together with more practical questions such as quantum key distribution, amplification and decoherence. Time symmetry and, especially, the two-state-vector formalism and weak values serve as a leitmotif passing throughout the issue. We have works discussing the foundations of determinism and collapse (Vaidman; Hofmann; Casher, Nussinov and Tollaksen; Richardson and Dowling) and works studying the applications of weak values (Lee and Tsutsui; Troupe; Lobo, Aharonov, Tollaksen, Berrigan, and Ribeiro). Continuing in the line of discussing open fundamental questions in quantum theory lies the paper of Aharonov, Cohen, Gruss and Landsberger which studies the appearance of decoherence and collapse in the framework of the two-state vector formalism. Finally, we have a third division of papers which deal with the Aharonov-Bohm effect, namely the papers of Nussinov, Nussinov, and Nussinov and Gray.

Vaidman's paper, "Quantum theory and determinism", challenges the consensus that quantum theory is the reason to believe that there is a genuine randomness in nature. Reviewing various approaches to interpretation of quantum mechanics, Vaidman argues that the universal wave function is the preferred ontology and, in his view, the best option is to take it as the only ontology. This brings determinism to the physical universe and removes any kind of action-at-a-distance from physics. It comes, however, at the price of introducing multiple parallel worlds which many physicists find hard to accept. Apart from extensive argumentation concerning the main thesis of deterministic quantum theory and analysis of the many-worlds interpretation, Vaidman provides several interesting and novel points about other interpretations. He provides an illuminating analysis of old and recent proposals for probabilistic 
models underlying outcomes of quantum experiments. Vaidman analyzes the meaning of old and new Bell-type arguments regarding nonlocality. He strengthens the GRW collapse theory by explaining why the "GRW tails" problem does not represent a weakness similar to the "empty waves" problem of the Bohmian interpretation. For the Bohmian interpretation, he presents an interesting option of a multiple Bohmian worlds interpretation.

The article by Holger F. Hofmann "Reasonable conditions for joint probabilities of non-commuting observables" investigates how the operator formalism of quantum mechanics represents the joint statistics of physical properties that cannot be measured jointly. Complementing the more detailed approaches to measurement statistics reported in other articles in this issue, the paper focuses on the general mathematical structure of Hilbert space, starting from the observation that the density operator representation of a state provides a simultaneous description of measurement probabilities for precise measurements of any two non-commuting observables, even though the measurements of these two observables can never be performed jointly. This formal similarity between quantum states and joint probabilities for pairs of non-commuting observables is then developed further by introducing logical requirements for the relation between the observable probabilities of individual measurement outcomes and the unobservable joint probabilities. Interestingly, this simple logical selection of quasi-probability representations narrows the choice down to the expectation values of projection operator products - an indication that the formalism itself might tell us much more about the relation between physical properties than textbook introductions suggest. The article thus illustrates well how a closer look at the mathematical structure of quantum theory can result in new insights into foundational problems of quantum mechanics.

The paper "Collapses and avoiding wave function spreading" by Casher, Nussinov and Tollaksen starts by describing the collapse issue in quantum mechanics which is connected with the apparent irreversibility of the chaotic imprints left by the interaction of the quantum system with the measurement of a complicated classical system. It then addresses the "inverse" issue of having an isolated many-particle quantum, yet a semi-classical system evolve into a completely spread out purely quantum system.

The next paper, "On the uncertainty of the ordering of nonlocal wavefunction collapse when relativity is considered," by Richardson and Dowling addresses the issue of the Lorentz covariance of a possible collapse of the wavefunction. They show that a description of the reduced density operator for Bob's photon displaying the uncertainty of Bob's measurement time (as measured by Alice's clock) is a function of the relative velocity of Alice and Bob's frames. Alice can then use this to calculate the measurement statistics that Bob will observe for the collapse time of his photon. These statistics indicate Alice will observe that the uncertainty in Bob's collapse time (in her frame) will always exceed the relativistic time difference between her clock and Bob's clock. Therefore, Richardson and Dowling argue that Bob's observed measurement time can never conclusively demonstrate a situation in which their time ordering for the state collapse will disagree.

Lee and Tsutsui's paper, "Merit of amplification by weak measurement in view of measurement uncertainty," showed the advantage of the weak-value amplification under noisy environments even though other researchers had found no advantage of the weak-value amplification from the viewpoint of the parameter estimation. Lee and Tsutsui mathematically formulate several noise sources, which are called an uncertainty in their paper, and calculate a signal-to-noise ratio. Their results reveal a new direction concerning the issue of weak-value amplification.

The paper "Quantum key distribution using sequential weak values" by James E. Troupe applies some of the uniquely quantum aspects of weak values to one of the central problems of the Internet age: secure communication. In this paper, Troupe proposes a new type of quantum key distribution in which the presence of an eavesdropper is determined by the effect such an eavesdropper has on the weak value of path information inside of a Mach-Zehnder (MZI) interferometer. Interestingly, the bits of the secure key are encoded and carried by the sequential weak value of a particular path through the double MZI setup. Bob is provided access to these bits via the correlations between his and Alice's post-selected weak measurement results. This approach does not obviously rely on the no-cloning theorem, and so may be another example of weak measurement providing a new resource, this time in quantum information. While the key generation rate currently possible with this technique is quite low, it is a promising beginning.

The next paper in this collection: "Weak values and modular variables from a quantum phase-space perspective" by Augusto C. Lobo, Yakir Aharonov Jeff Tollaksen, Elizabeth M. Berrigan and Clyffe de Assis Ribeiro provides 
an alternative approach to the same underlying question proposed by Hofmann. It also reviews various approaches to phase space quantum mechanics. Each approach enhances the mathematical understanding of certain aspects of weak values and modular variables. For instance, a unified phase space treatment of the measuring system shows in a very clear manner that both the real and imaginary parts of the weak value have equal physical status. In addition, an opposite approach where one examines the geometric structure of the measured system exhibits many interesting properties of the weak value concept. In particular, the weak value of the state of a single qubit is shown to be nothing else, but the the complex coordinate obtained through the stereographic projection of its Bloch sphere representation. Finally, an approach to describe the modular variable exchange between particles and the slit apparatus in quantum interferometry is discussed within Schwinger's finite quantum kinematical formalism. It remains uncertain, though, how to extend this mathematical structure to incorporate the interaction with the electromagnetic field and to add geometries that are more general. In particular, it seems to be very hard to extend these ideas to energy-time modular variables in a covariant manner. Yet, one may expect that this approach may shed light on the differences between dynamical non-locality and the more commonly known kinematic non-locality exhibited by entangled quantum states.

The next paper by Aharonov, Cohen, Gruss and Landsberger, "Measurement and collapse within the two-state vector formalism", studies the appearance of decoherence and collapse in the framework of the two-state vector formulation of quantum mechanics. Like Vaidman's paper it discusses the many-worlds interpretation, but shows that it is possible to construct a final boundary condition, which encodes all measurement outcomes and permits time reversal, even within a single branch of the universe. Together with the work of Casher, Nussinov and Tollaksen, which further analyzes collapse and extends the above results to semi-classical WKB states, a novel approach towards resolving the measurement paradox is presented in the form of a well-defined boundary between classical and quantum regime.

Next is the paper "The notion of a random gauge and its interpretation" by John E. Gray of the Navy. The idea of a gauge first emerged in the ninetieth century when Lorentz, the minor one, introduced the idea of a gauge to decouple Maxwell's equations, which then could be solved by separation of variables. Throughout the twentieth century, whenever a new instance of a gauge has appeared, it has heralded new and interesting physics. In quantum theory, it has proven to be a key concept both in understanding the forces between particles and in how interactions can occur without an external force (the so-called A-B effect and its generalization to Berry phase). Gray has suggested two different notions of gauge by suggesting the means by which it may be treated randomly. He then suggests some means to experimentally verify the notion. While this concept is novel, it remains to be seen whether it will prove fruitful. Time will tell.

Finally, the paper "Decoherence due to thermal effects in two quintessential quantum systems" by Nussinov, Nussinov and Nussinov addresses the important issue of quantum decoherence induced by finite temperatures. Specifically they show how such decoherence effects can exponentially weaken two effects which are of a genuinely quantum mechanical nature. The first is the angle dependent/polarization-driven Casimir force and the second is the topological force between normal fluxons in a super-conductor induced by the Aharonov-Bohm phases picked up by unpaired electrons circulating between the fluxons. 